\title{
Adaptive Multi-Cost Routing Protocol to Enhance Lifetime for Wireless Body Area Network
}

\author{
Muhammad Mateen Yaqoob ${ }^{1}$, Waqar Khurshid ${ }^{1}$, Leo Liu' ${ }^{2}$, Syed Zulqarnain Arif ${ }^{1}$, Imran Ali Khan ${ }^{1}$, \\ Osman Khalid ${ }^{1, *}$ and Raheel Nawaz \\ ${ }^{1}$ Department of Computer Science, COMSATS University Islamabad, Abbottabad Campus, 22060, Pakistan \\ ${ }^{2}$ Department of OTEHM, Manchester Metropolitan University, Manchester, UK \\ *Corresponding Author: Osman Khalid. Email: osman@cuiatd.edu.pk \\ Received: 31 October 2021; Accepted: 24 December 2021
}

\begin{abstract}
Wireless Body Area Network (WBAN) technologies are emerging with extensive applications in several domains. Health is a fascinating domain of WBAN for smart monitoring of a patient's condition. An important factor to consider in WBAN is a node's lifetime. Improving the lifetime of nodes is critical to address many issues, such as utility and reliability. Existing routing protocols have addressed the energy conservation problem but considered only a few parameters, thus affecting their performance. Moreover, most of the existing schemes did not consider traffic prioritization which is critical in WBANs. In this paper, an adaptive multi-cost routing protocol is proposed with a multi-objective cost function considering minimum distance from sink, temperature of sensor nodes, priority of sensed data, and maximum residual energy on sensor nodes. The performance of the proposed protocol is compared with the existing schemes for the parameters: network lifetime, stability period, throughput, energy consumption, and path loss. It is evident from the obtained results that the proposed protocol improves network lifetime and stability period by $30 \%$ and $15 \%$, respectively, as well as outperforms the existing protocols in terms of throughput, energy consumption, and path loss.
\end{abstract}

Keywords: Multi-cost routing protocol; network lifetime; wireless body area network; adaptive routing; residual energy; clustering; poisson distribution

\section{Introduction}

Recent advancements in information and communication technologies have enabled new opportunities to facilitate people in various domains of life [1]. One such area that has seen significant development, especially after the outbreak of COVID-19 is remote healthcare. Distant health monitoring is an evolving technology that enables healthcare providers to observe, diagnose, and prescribe patients without the need of their physical presence [2]. In recent years, Wireless Body Area Network (WBAN) has emerged as a most promising communication technology for remote healthcare that enables applications to monitor a patient's health with the help of sensor nodes [3]. WBAN consists of different kinds of wirelessly connected sensor nodes implanted or surface mounted on a human body. The 
primary aim of WBAN sensors is to extract various physical parameters, such as body temperature, pulse rate, respiratory measurement, sugar level, or amount of calories burnt in a workout. The sensor nodes in a WBAN can be deployed in different topologies such as a star, mesh, or tree, depending upon the specific application requirement. The data collected by the sensors is transferred to a remote server node for processing and analysis, and/or to generate alerts if some abnormality is detected. The use of WBANs is not just limited to healthcare, but they also have applications in other domains as well, like multimedia, gaming, and sports etc. [4]. The WBAN can be used to monitor health of an athlete during training, or it can be used to monitor the mental condition of a gamer. WBAN devices can also be used for video capturing during internal body scans/imagery.

In medical field, the use of WBAN allows a patient to not stay confined to a single location tethered with wires and monitoring devices. Rather, a patient can move freely, or may leave hospital facility while his/her vital signs (e.g., body temperature, pulse rate, respiration rate, blood pressure, etc.) are constantly being monitored and reported. This helps in improving the quality of life of the patient. Moreover, when the patient's data is collected in a natural environment over a longer period, this provides more useful information, allowing for accurate diagnoses and treatment [5]. A typical organization of WBAN is shown in Fig. 1.

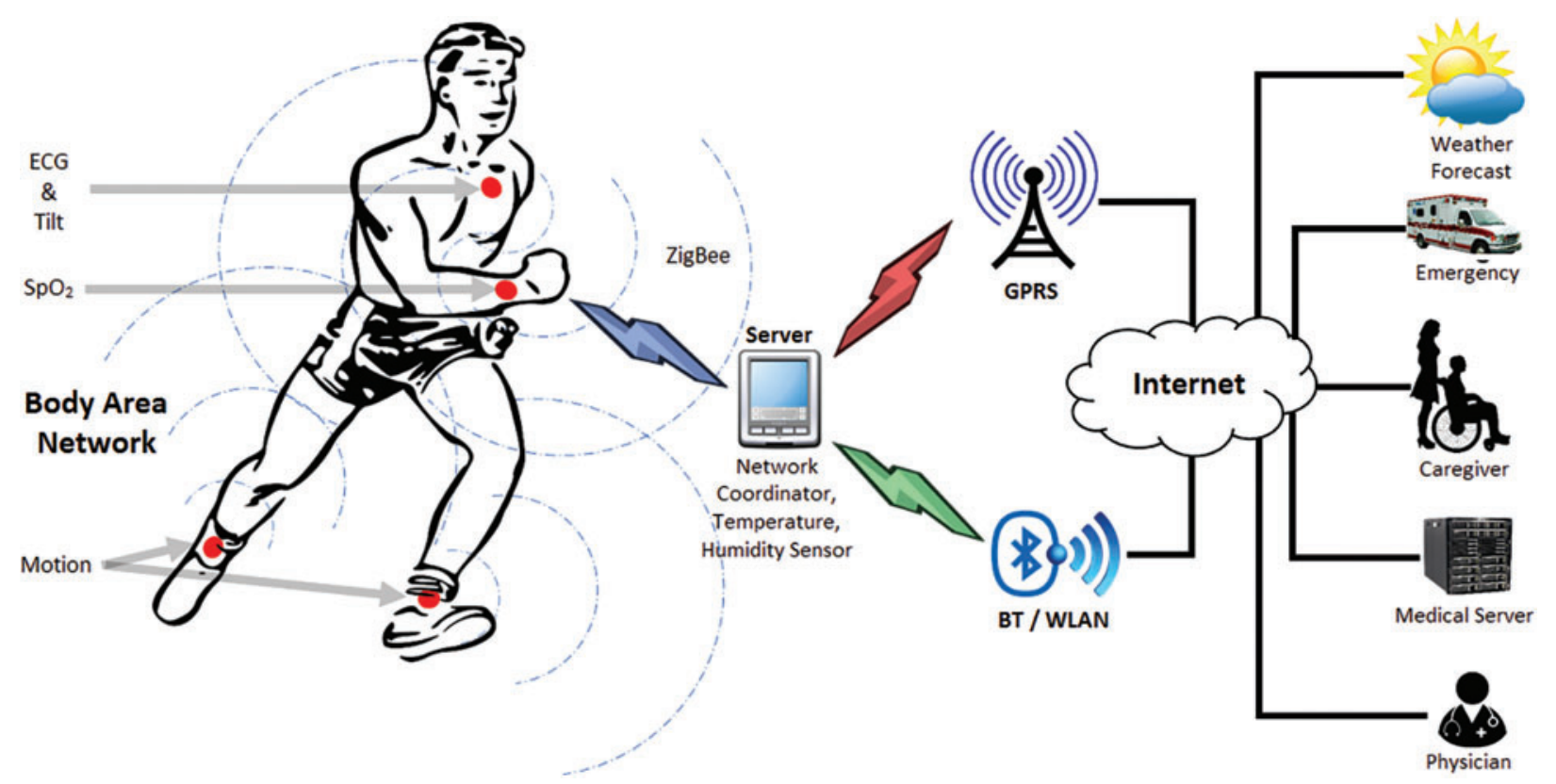

Figure 1: Architecture of WBAN

Despite many benefits, WBANs suffer from several limitations and challenges due to miniaturized nodes with shorter battery lives. Smart health application using WBAN requires seamless network connectivity and autonomous self-organizing capabilities of inter-connected sensor nodes [6]. Due to resource limitations in terms of battery power, available bandwidth, transmission power, buffer size, and processing capacity, the traditional routing protocols designed for Wireless Sensor Networks (WSNs) are not appropriate for WBANs [7]. Moreover, the movement of human body, and heterogenous nature of nodes with different battery lifespans may result in link failures, creating network instability. Therefore, routing, and medium access become challenging in such unstable conditions. The routing protocols for WBANs need to be designed carefully to be energy efficient and robust 
[8]. The sensed data from patient contains diverse and critical information like Electrocardiogram (ECG) and heart rate, along with other information such as temperature and movement. Therefore, the prioritization of data based on criticality for timely delivery is an important task that must considered by an efficient routing protocol.

Several routing protocols for WBAN have been proposed in the past, designed for different application scenarios [3,4]. Generally, the WBAN routing protocols are classified as temperaturebased, posture-based, cross-layer, cluster-based, and QoS based [8], depending upon the application they are targeting. For instance, Nadeem et al. [9] proposed a multi-hop routing protocol for link efficiency in WBAN.

The authors considered the residual energy of the nodes for improving the link efficiency in WBANs. However, they did not consider routing with multi-cost function. Moreover, the authors evaluated the performance of their proposed method with limited number of sensor nodes. In [10], energy efficiency for multi-hop communication is improved using a linear programming-based routing protocol. However, the authors did not consider network lifetime as a parameter for performance evaluation. Moreover, their proposed protocol lacks multi-cost routing function. Distance-based routing protocol for multi-hop communication in a hospital-based patient monitoring application is proposed in [11] to improve energy efficiency. The proposed protocol is evaluated with a limited number of sensor nodes and considered only distance as the route cost function. In [12], the authors proposed a multi-chain routing to estimate the sink mobility and improve the energy efficiency.

As we can observe from the aforementioned discussion that most of the existing works did not consider the network lifetime as an evaluation criterion for their protocols, and they have performed simulations by considering a limited number of sensor nodes. Moreover, the protocols did not consider adaptive routing, used single attribute to calculate route cost function, and provided evaluation with limited number of nodes. In this paper, we propose a routing protocol for WBANs that provides adaptive routing using a multi-cost function and involves many nodes to collect variety of sensory data from a patient. The proposed protocol works for normal as well as event driven traffic. The multi-cost routing metric comprises of minimum distance from the sink, nodes' temperature, priority of sensed data, and maximum residual energy of the sensor nodes. We perform strategic sink placement which helps in effective aggregation of traffic from every sensor node and makes it more discoverable $[9-10,13,14]$. The proposed protocol improves the network stability and lifetime. The following is the summary of our contributions:

- An adaptive routing protocol is proposed based on multi-cost function including maximum residual energy of nodes, temperature of sensor nodes, priority of sensed data, and minimum distance from the sink.

- A traffic model based on Poisson distribution is developed to generate traffic.

- Traffic prioritization is modelled using a weighted approach.

- Strategic sink placement is discussed for improved data aggregation.

- The proposed scheme is evaluated for the parameters: network lifetime, stability period, throughput, energy consumption, and path loss for the sensory data obtained from patients.

The rest of the paper is organized as follows. Section 2 presents the literature review. Section 3 explains the proposed work. Performance evaluations and results are discussed in Section 4. Finally, Section 5 concludes the paper. 


\section{Literature Review}

WBAN is an evolving technology with newer, miniaturized products, constantly being introduced into the market. The routing protocols play an important role in the design of low cost, energy-efficient, and reliable WBANs. In the existing literature, there is no standardized categorization of WBAN protocols. However, based on the application scenarios and design objectives, the routing protocols for WBANs can be generally classified as postural-based, temperature-based, cluster-based, crosslayer, energy-aware, and QoS-based [8]. In the subsequent text, we briefly present a comparative study of state-of-the-art WBAN routing protocols.

In [9], the authors proposed a protocol, named SIMPLE to route information in WBANs. The proposed protocol utilizes a cost capacity function to choose a suitable route towards the sink. Cost function is determined based on the distance among the sensing nodes and the number of hops from the sink. A sensor with least cost function is chosen as a parent node. In the SIMPLE routing protocol, forwarding node is selected based on minimum distance to the sink. Each forwarding node is responsible for data aggregation from its neighboring nodes and to forward the aggregated data towards the sink. The authors used Time Division Multiple Access (TDMA) for scheduling the transmission of sensed data among the sensors. The proposed protocol divides each TDMA cycle into the following sub-cycles: (a) controller sub-cycle and (b) information sub-cycle. The issue with SIMPLE is that it only considers minimum distance as the routing criteria at the cost of higher energy consumption. Javaid et al. [13] proposed an improved version of SIMPLE, named as iM-SIMPLE. They used integer linear programming to improve the throughput and reliability of WBAN.

In [10], the authors proposed M-ATTEMPT, which is an energy and temperature-aware routing protocol for WBANs. Their proposed protocol reduces energy consumption for high traffic load using biosensors. According to the M-ATTEMPT protocol, random placement of sensing nodes is accomplished by using descending order of data rate. A sink is placed at center of patient's body. The authors used mobile sensor nodes to collect data from the patient's body. Moreover, linear programming is used to optimize energy management of nodes. In [11], the authors proposed a routing protocol named as Distance Aware Relaying Energy efficient (DARE) for multi-hop body sensor networks. The protocol considers minimum distance among the relaying sensor nodes as the routing metric. The authors evaluated the performance of the protocol under different topologies. Moreover, the authors considered an emergency clinic scenario and used seven sensors including a Body Relay (BR) to continuously receive information and event driven data from the patient. The sensors record various vital parameters associated with a patient's health which includes ECG, heart rate, pulse, temperature, glucose level, and movement. DARE uses a single metric-based routing which can lead to insufficient information for effective routing in WBANs.

Jafri et al. proposed a power efficient protocol, named as PEGASIS [12]. The authors proposed a mobility mechanism for the sink node to improve energy efficiency in routing. To enhance the performance of WBANs for a mobility supported network, a deep learning-based routing technique is proposed in [15]. The proposed scheme uses deep learning and Adam optimizer to estimate the position of mobile sensor nodes. The protocol aimed to improve the network lifetime for heterogeneous and homogeneous network configurations. However, the protocol only considers the estimation of sensor movement as the routing metric, which can lead to decline in the energy efficiency of the protocol. There are several routing algorithms available for healthcare application using WBANs. An energyware routing protocol is designed in [16] for hospitals that performs best route selection to transmit data packets. The authors proposed an energy-aware stable routing scheme that is able to derive benefit from the residual energy and stability of nodes. Jamil et al. developed an adaptive thermal-aware 
routing protocol to solve the existing thermal issues [14]. They proposed a multi-ring approach that selects alternate end-to-end routes when the temperature of a node at any specific route exceeds a threshold value. The partitioning of the network into multiple rings and maintaining ring information along with temperature and hop count across the network results in increased overhead that is not preferable method for resource limited WBANs. Moreover, the protocol is not adaptable to body movements.

Chavva et al. proposed an energy efficient multi-hop routing protocol for data transfer between sensor nodes and sink [17]. For optimal route selection, they used the Mamdani fuzzy logic [18]. The authors used five fuzzy rules based on the residual energy level. All the data transmission is scheduled using TDMA time slots. However, considering a single metric for data routing is not efficient. Moreover, TDMA scheduling is not suitable for emergency data transmission [4]. Aadil et al. proposed a routing protocol based on clustering for remote monitoring of a patient's health [19]. Their proposed method intended to optimize the cluster head $(\mathrm{CH})$ selection process and for this purpose, they used three optimization algorithms, namely, the ant colony optimization, multiobjective particle swarm optimization, and the comprehensive learning particle swarm optimization. The optimal selection of $\mathrm{CH}$ improved the overall routing performance. However, their proposed protocol is not evaluated under high density of sensor nodes and for various traffic types. The summary of discussed related work is shown in Tab. 1.

\section{Design of Proposed Protocol}

\subsection{Radio Model}

There are various radio models proposed by researchers to examine communication and energy characteristics amongst the sensing nodes [20,21]. Our proposed work is inspired by the model presented in [20] due to the following two reasons: (a) it has lower complexity and (b) it depicts the communication scenarios that are closely related to WBANs. The expressions for data transmission energy costs, receiving costs, and amplification costs [20] are presented as follows.

$E_{T_{x}}(k, d)=E_{T_{x} \text { elec }} \times k+\left(\varepsilon_{\text {amp }} \times k \times d^{2}\right)$.

$E_{R_{x}}(k)=E_{R_{x} \text {-elec }} \times k$.

Here, $d$ is the distance between transmitter and receiver. The parameters $E_{T_{x}}$ and $E_{R_{x}}$ are the energy consumption per packet costs of the transmitter and receiver, respectively. Similarly, $E_{T_{x}-e l e c}$ and $E_{R_{x}-\text { elec }}$ are the per bit energy consumption values for the transmitter and receiver electronics circuitries, respectively [22]. The parameter $\varepsilon_{\text {amp }}$ is the radio amplifier type and $k$ is the packet length. The loss coefficient $\eta$ is different for human body as compared to terrestrial networks, so we rewrite Eq. (1) in terms of $\eta$ as:

$E_{T x}(k, d, \eta)=E_{T x-\text { elec }} \times k+\left(\varepsilon_{\text {amp }} \times \eta \times k \times d^{\eta}\right)$.

We utilized Nordic nRF 2401A [23] because it is a low power transceiver and uses the free-licensed band of 2.4 GHz. The details of Nordic nRF 2401A radio transceiver are described in Tab. 2. 





Table 2: Radio parameters of Nordic nRF 2401A

\begin{tabular}{ll}
\hline Radio Parameters & Value \\
\hline DC Transmission (mA) & 10.5 \\
DC Receiving (mA) & 18 \\
Power supply (V) & 1.9 \\
$E_{T_{x} \text {-elec }}($ nanoJ/bit) & 16.7 \\
$E_{R_{x} \text {-elec }}(\mathrm{nanoJ} / \mathrm{bit})$ & 36.1 \\
$\varepsilon_{\text {amp }}$ (nanoJ/bit/mn) & 1.97 \\
\hline
\end{tabular}

\subsection{Path Loss Model}

The path loss is measured in the form of decibels $(\mathrm{dB})$ and it signifies the signal attenuation [24]. It is notable that the signal power is corrupted by a factor called Additive White Gaussian Noise (AWGN).

Path loss is defined as a variance between transferred power and received power of any device. However, antenna gain is not mandatory to be considered. In the field of WBAN, movement of human body, dissimilar body positions, hands, clothes, and other accessories disturb the transferred signal. Path loss associated with distance and frequency is stated as given in [24].

$\mathrm{PL}(\mathrm{f}, \mathrm{d})=\mathrm{PL}_{\mathrm{o}}+10 \mathrm{n} \log _{10}\left(\frac{\mathrm{d}}{\mathrm{d}_{\mathrm{o}}}\right)+\mathrm{X} \sigma$.

In Eq. (4), the $P L_{\mathrm{o}}$ represents received path loss, $\mathrm{d}$ indicates the space among transmitter and receiver and $d_{o}$ is considered as referenced distance. Moreover, the value of $n$ relies on propagation environment, and it is the path loss coefficient. If there is a free space communication, then the value of $n$ will be 2 , but for communication in the case of WBAN, the value of $n$ ranges from 3 to 4 for line of sight (LOS) communication, and between 5 and 7.4 for a non-line of sight (NLOS) communication. Gaussian random variable is denoted by $X$, and $\sigma$ denotes the standard deviation. The parameter $P L_{0}$ indicates the path loss at reference distance $d_{o}$ so it is expressed as:

$$
P L_{O}=10 \log _{10}\left(\frac{[4 \pi d f]^{2}}{c}\right) \text {, }
$$

where the parameter $f$ indicates frequency, $d$ is the distance (between the transmitter and receiver), $c$ represents the speed of light, and the value of reference distance $d_{o}$ is $10 \mathrm{~cm}$. The standard deviation variable $X_{\sigma}$ is used for predicting the signal strength between boundaries of the transmitter and receiver.

\subsection{Adaptive Multi-Cost Based Routing Protocol (AMCRP)}

We assume that different multipurpose biosensors are placed on a patient's body. We placed 19 biosensors and 5 relay nodes on the body. These biosensors are placed on appropriate sensing location across the whole body of the patient as shown in the Fig. 2. After several empirical trials, the best location for the sink node placement is found to be the center of a patient's body. Similar placements are performed by the authors in $[9-10,13,14]$, as this allows effective data aggregation by making the sink more discoverable to the sensor nodes. The ECG, EMG, glucose, and blood pressure sensors are 
assigned higher priority, and the traffic from these sensors is considered as critical data traffic [25,26]. Our proposed routing protocol is adaptive in nature and capable of working in critical and normal data traffic scenarios. Moreover, we extend the performance by considering a multi-cost routing metric consisting of minimum distance from the sink, temperature of sensor nodes, priority of sensed data, and maximum residual energy of the sensor nodes. Details of our proposed work are in the following subsections.

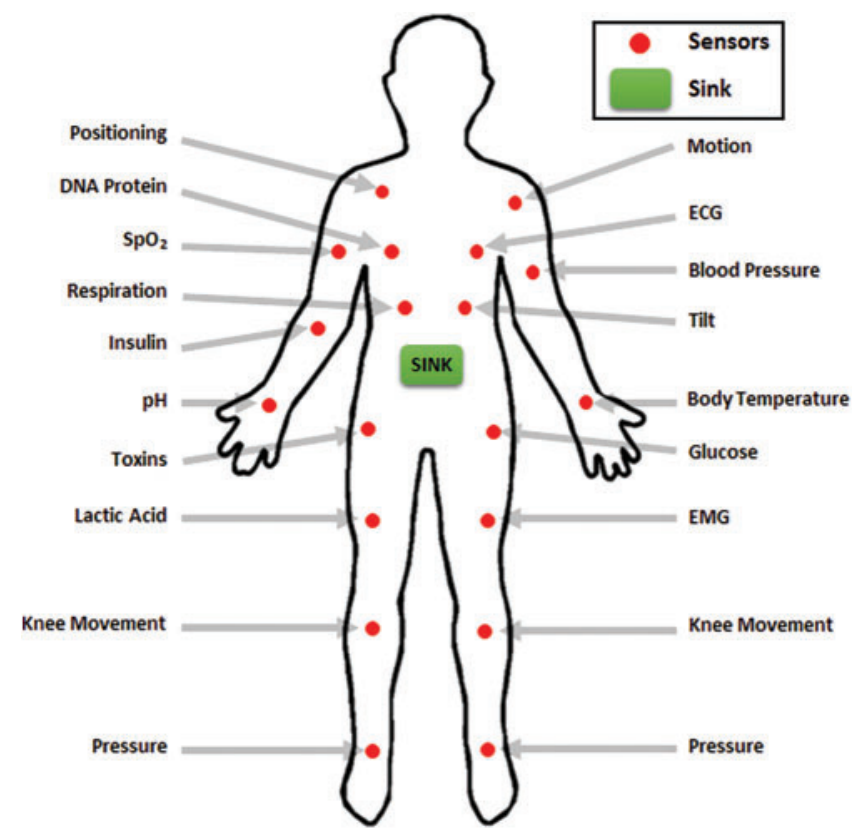

Figure 2: Configuration of sensors and sink node on human body in proposed AMCRP

\subsubsection{Initialization Phase}

The sink broadcasts a packet which contains its location. Each receiving sensor stores the location of the sink and then rebroadcasts a packet which contains sensor ID, current temperature of the node, residual energy status, priority of sensor, and its location. In this manner, all the sensors are updated with the location of the neighbor nodes and the sink [27].

\subsubsection{Routing Cost Function}

To reduce energy consumption and maximize network lifetime, we utilized multi-hop communication for WBANs [28]. Our proposed protocol selects new relay node for data routing and forwarding in each round of communication. In the initialization phase, the sink node receives sensor ID, location, priority, and residual energy of each relay and sensor node.

On having this information, the sink calculates the routing cost for every relay node, and then disseminates this cost to every other relay node. Based on the cost function, every relay node decides whether to become forwarder node or not. Let the ID of a sensor node be represented as $j$, then the routing cost function $R C F(j)$ is calculated as:

$$
R C F(j)=\left(\frac{\mathrm{D}}{\operatorname{RE}(\mathrm{j}) \times \operatorname{temp}(\mathrm{j})}\right) \times \operatorname{Pr}(j) .
$$


Here, $D$ the distance of node $j$ from the sink node, $R E(j)$ is the residual energy of the sensor node computed as $R E(j)=E_{n}-E_{c}$, where $E_{n}$ is the total energy of the sensor node and $E_{c}$ is the consumed energy. The parameter $\operatorname{Pr}(j)$ is the priority of sensor node and it has value of 2 for high priority traffic nodes and 1 for other nodes whereas $\operatorname{temp}(j)$ is the current temperature of the sensor node $j$. After the $R C F(j)$ is computed, the sink selects the appropriate forwarding node with minimum route cost function. Sink will calculate $R C F$ and notify all the relay nodes about $R C F$ after it will elect the forwarder node. This will be done to notify the sensing nodes about their forwarder (relay) node so that they can effectively transmit the sensed data towards the sink. Eventually, all the neighbor sensors send data to the aforementioned forwarder node which then aggregates the data and transmits it to the sink. The selected forwarder node has minimum distance to the sink and maximum residual energy. Consequently, this node is better selection for data routing within the available resources. Algorithm 1 presents the steps involved in candidate node selection process.

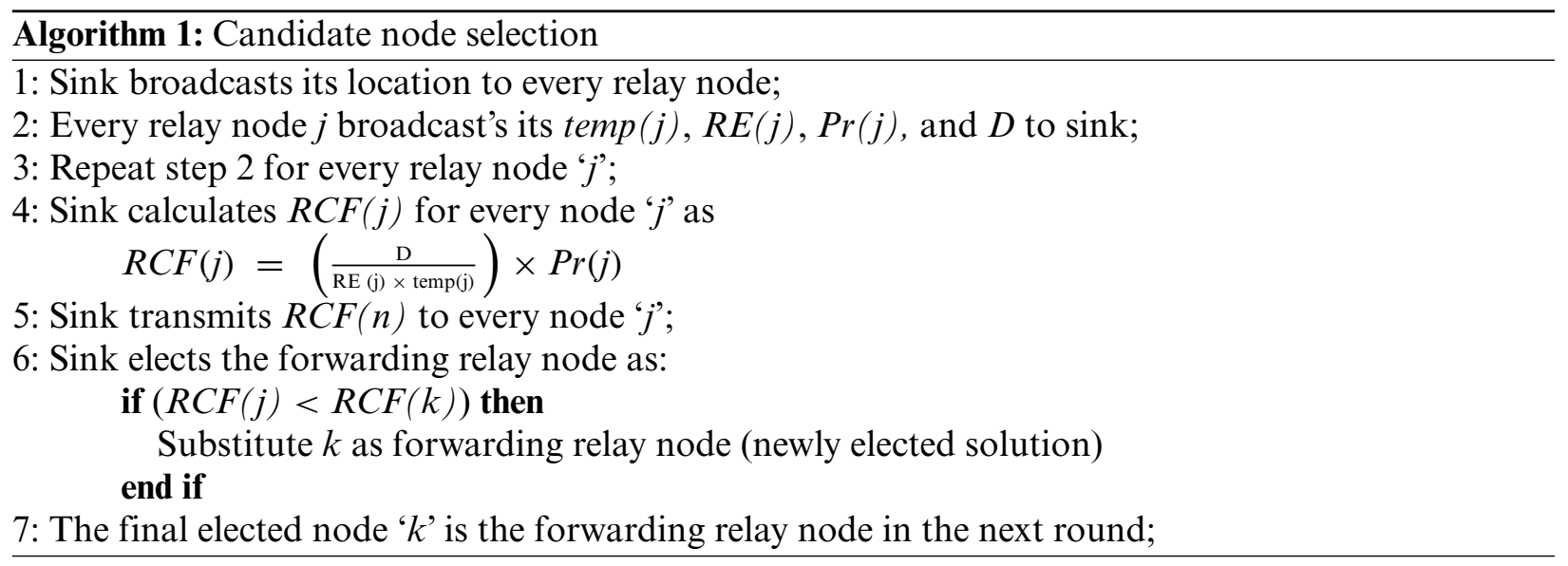

\subsubsection{Data Transmission and Scheduling}

The main objective of the proposed protocol is to minimize the energy consumption in order to enhance network lifetime of WBAN. To make the protocol workable for both normal and critical data traffic situation in WBAN, we have assigned the priorities for different types of packets. The sensors such as ECG, EMG, glucose, and blood pressure are assigned higher priority, and the traffic from these sensors is considered as critical data traffic.

In this phase, sink assigns time slots based on Priority-Based Enhanced Time Division Multiple Access (PTDMA) protocol proposed in [29] to the forwarding node and to the other sensor nodes. The sensor nodes disseminate the sensed data to the forwarding node within the scheduled time slots and the forwarding node aggregates the data and transmits it to the sink in the allocated time slot. When a node has no data to transmit, then it switches to idle mode. Only at their transmission time slot, the nodes wake up. Hence, this PTDMA time scheduling of sensors reduces nodes' energy consumption which lead to improvement of network lifetime. Communication flows from sensor-to-sink, or from sensor-to-sensor and then to sink, but communication outside the WBANs is not considered in this protocol. Forwarded node is selected based on multi-cost function $R C F(j)$.

When a sensor node has sensed the data under event driven traffic scenario, it specifies the occurrence the event based on the threshold, and it varies from patient to patient [30,31]. Therefore, it is essential to mathematically model the probability of occurrence of each event taking place in 
the sensor node. For any data transmission event under normal traffic condition, the frequency of occurring events, $S$ is computed using the Poisson distribution [32]:

$\mathrm{P}(\mathrm{S})=\frac{\lambda^{\mathrm{k}} e^{-\lambda}}{k !}$.

where $\mathrm{P}(\mathrm{S})$ is the computed frequency of occurring events using Poisson distribution and $\lambda$ is the mean number of events happening at that point distribution of frequency. In our proposed system, we assume that the WBAN comprises of multi-hop, multi-frequency bands, and multiple paths. Therefore, a certain weight is assigned to every sensor node. Our proposed AMCRP protocol has the following key attributes:

- Each network node is fixed in its location.

- Routing is adaptive and it is done using a multi-cost function $R C F(j)$ consisting of residual energy, temperature of sensor nodes, distance to the sink, and priority of data traffic.

- The sink node is placed at the middle of the body liable for data gathering of all sensor nodes.

- Sink is assumed to have greater power resource and computational capabilities.

- Individual nodes transmit their data using PTDMA.

- Every individual sensor may transmit data through a forwarder node to a destination node i.e., sink node.

The simulation parameters for our AMCRP protocol are summarized in Tab. 3.

Table 3: Simulation parameters

\begin{tabular}{ll}
\hline Parameter & Value \\
\hline Simulation area & $2 \mathrm{~m} \times 2 \mathrm{~m}$ \\
Number of sensor nodes & 19 \\
Number of relay nodes & 5 relay nodes \\
Sink & 1 static \\
Type of devices & Body Sensors, Body Relay, Sink \\
Energy parameters & $E_{o_{B S s}}=0.5 \mathrm{~J}$ \\
& $E_{o_{B R}}=1 \mathrm{~J}$ \\
Communication type & $E_{o_{S i n k}}=$ infinite \\
Size of a packet & Multi-hop \\
\hline
\end{tabular}

\section{Results and Discussion}

For the evaluation of the proposed AMCRP protocol, we conducted a simulation consisting of 10,000 rounds using MATLAB simulator. We used 19 sensor nodes and 5 relay nodes which are randomly distributed and sink node is placed in the center of the network. We studied the performance of AMCRP and compared it with the existing protocols SIMPLE and M-ATTEMPT. Primarily, all of the sensor nodes have limited energy sources $(0.5 \mathrm{~J}$ per node for sensing nodes and $1 \mathrm{~J}$ per node for relaying sensors). When communication among sensor nodes takes place, the energy is depleted. The transmissions are ceased whenever the energy sources are vanished. The following are the parameters and their meanings used in the current section. 
- Stability period: It is the time period of network operation till the first node dies. The onward time period is considered as unstable period [10].

- Network lifetime: Time period until all of the sensor nodes in a WBAN die.

- Residual energy: It is the remaining energy of the node after each round of communication.

- Throughput: It is termed as the total number of successfully received packets at the sink node.

- Path loss: It is defined as the reduction in power level when the data signal is transmitted from source to destination, and it is measured in decibels (dB). Path loss plays a key role in the analysis and design of link budget for practical implementation.

Fig. 3 shows the comparisons for network lifetime. In the worst case, the stability period of AMCRP is better than SIMPLE and M-ATTEMPT that have stability periods of 3000 and 1500 rounds, respectively, as compared to AMCRP that has a stability period of 3500 rounds of communication. AMCRP performs better in terms of network lifetime because in case of M-ATTEMPT and SIMPLE, all the sensor nodes die after 7000 rounds but in the case of AMCRP protocol only 4 nodes die. In M-ATTEMPT, when temperature of forwarder node rises, the node selects alternate longer path that consumes more energy. Hence, these nodes die early. In SIMPLE, nodes die early due to continuous and single hop transmission. Comparatively, the proposed AMCRP achieves 15\% more stability period and 30\% longer network lifetime.

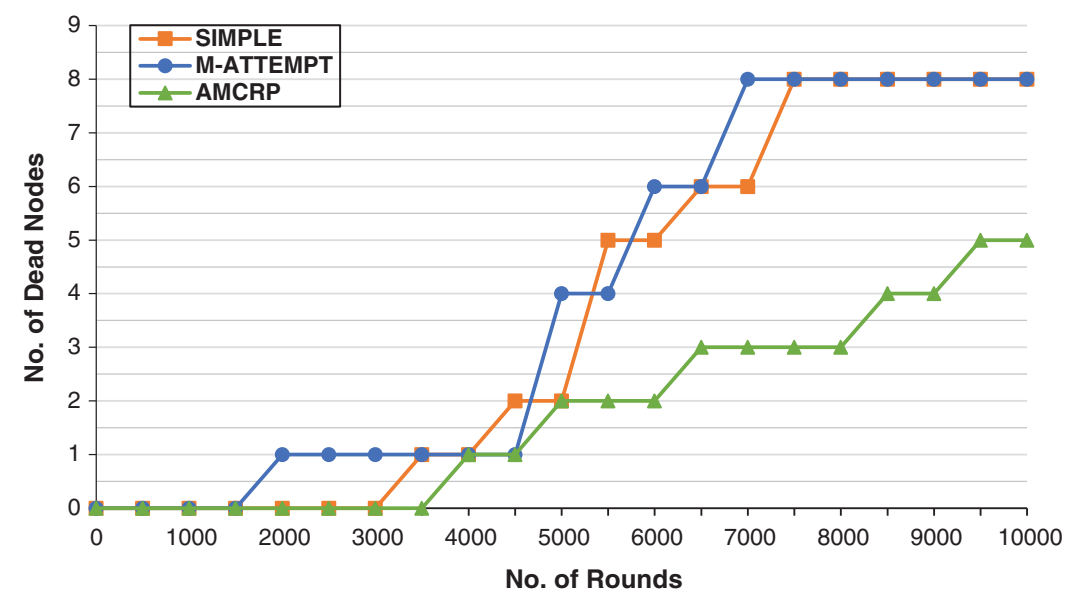

Figure 3: Network lifetime analysis

As discussed earlier, WBAN transfers critical and time sensitive data of patients, and due to this it requires a protocol with minimal packet drop ratio. As reflected in Fig. 4, AMCRP attains high throughput compared to M-ATTEMPT and SIMPLE. This is due to the energy conservation property of AMCRP that enables maximum number of nodes to stay alive, thus more nodes contributing to the message transfer, and thereby increasing the throughput. The proposed protocol has a multi-cost route computation function $R C F(j)$ that allows it to send maximum number of packets, with minimum energy consumption. The stability period of M-ATTEMPT and SIMPLE is smaller than AMCRP. This implies that they will send small number of packets to the sink, thus reducing the throughput. 


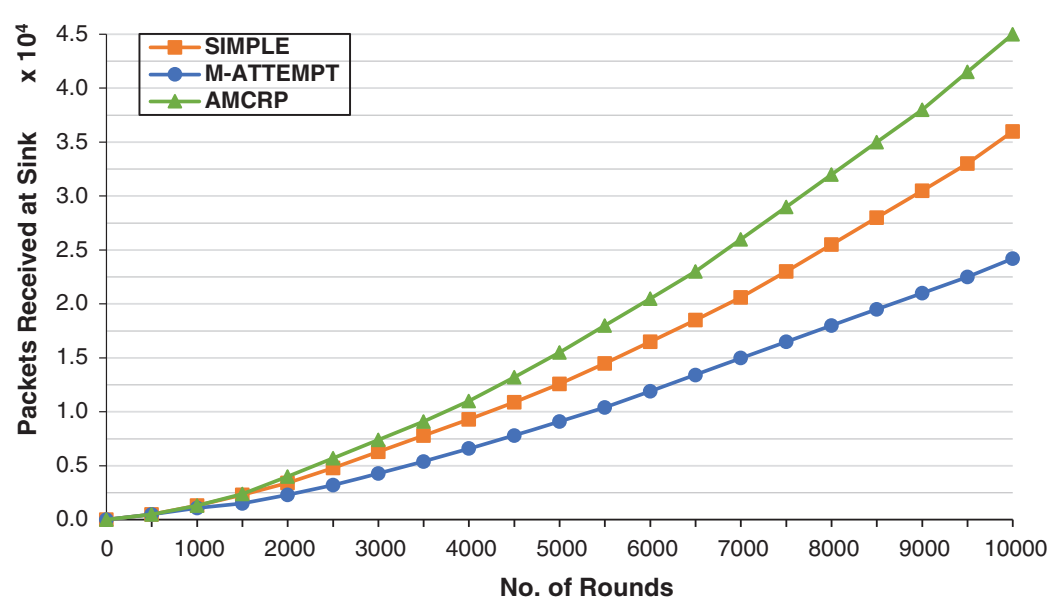

Figure 4: Throughput and packet received at the sink

Fig. 5 depicts the network energy consumed in each round of communication. The results indicate that AMCRP consumes 25\% lesser residual energy as compared to SIMPLE and M-ATTEMPT due to the energy-efficient route cost computation function. Fig. 6 shows the results of path loss, as a function of frequency calculated in Eq. (5) and distance. Our proposed protocol has reduced path loss as compared to M-ATTEMPT and SIMPLE protocols. This is because the path loss relies on the communication distance, and the proposed AMCRP protocol involves more nodes placed at shorter distances in a multi-hop communication compared to M-ATTEMPT and SIMPLE which eventually reduces the distance of communication and lead to lesser path loss.

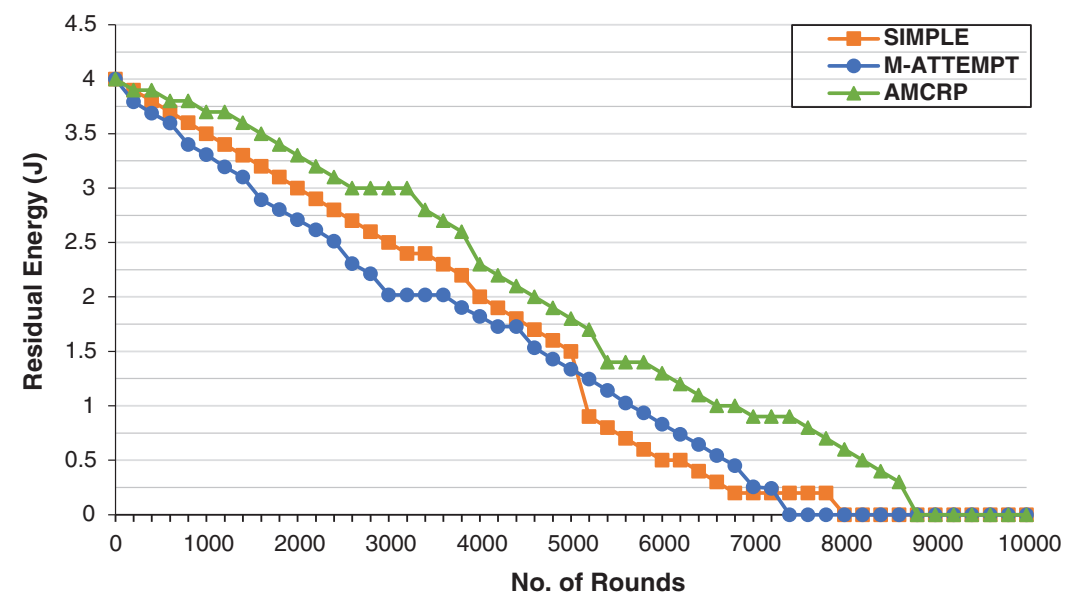

Figure 5: Analysis of remaining energy 


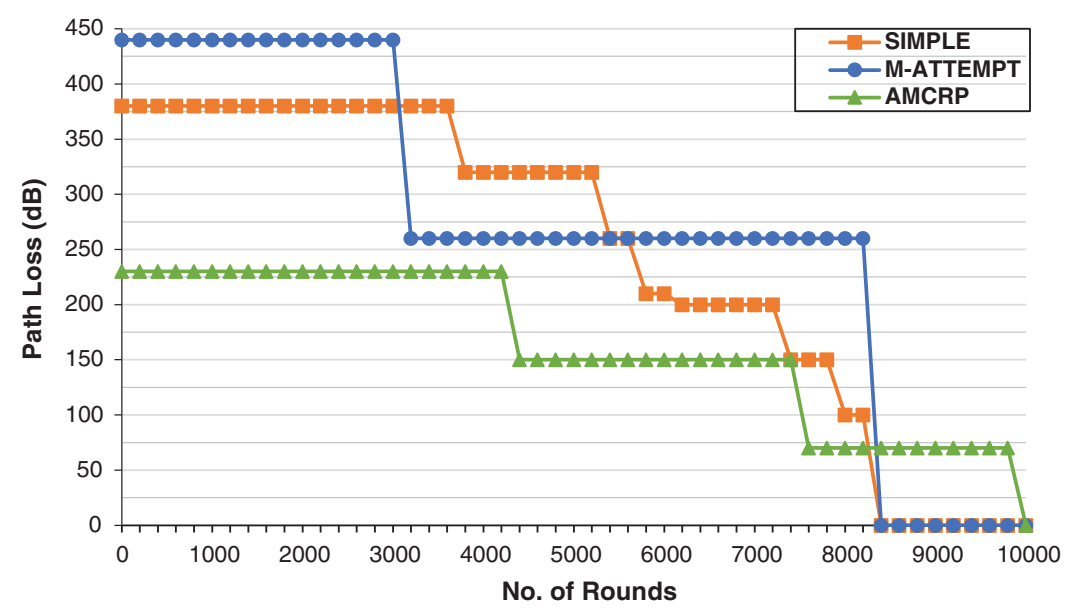

Figure 6: Analysis of path loss

\section{Conclusions}

In this paper, we proposed an energy-efficient adaptive multi-cost routing protocol for WBANs. Our main goal is to improve the network lifetime as well as to enhance the throughput and residual energy. To verify the correctness of the proposed protocol, simulations are performed using MATLAB. Various parameters are considered related to routing issues and the comparisons are made with existing baseline protocols M-ATTEMPT and SIMPLE. The simulation results indicated an improved performance of the proposed protocol over the baselines. The proposed AMCRP protocol supports normal traffic, multi-hop communication, enhanced network lifetime and stability period, and uses multi-cost route function. It is observed that the AMCRP protocol provides $25 \%$ more residual energy, achieves $15 \%$ more stability period and 30\% longer network lifetime. The proposed protocol provides enhanced network lifetime and higher stability period at the cost on increased processing overhead at relay nodes by isolating sensor nodes from participating in the election of new forwarder node. Although this helps in increasing the lifetime of sensor nodes, more number or relay nodes will be required to provide backup in case of energy depletion.

In future, we aim to improve the routing model under the effect of different physical phenomena, such as interference, noise, etc. for different mobility scenarios in WBANs. Additionally, we will employ various QoS metrics to further enhance the energy efficiency of the proposed scheme. Furthermore, we will focus on the reducing the processing overhead for relay nodes to further enhance the performance of the WBANs.

Acknowledgement: We are thankful to our colleagues who helped us in finalization of this paper.

Funding Statement: The authors received no specific funding for this study.

Conflicts of Interest: The authors declare that they have no conflicts of interest to report regarding the present study. 


\section{References}

[1] C. Liang, T. Baker, Y. Li, R. Nawaz and Y. -A. Tan, "Building covert timing channel of the IoT-enabled MTS based on multi-stage verification," IEEE Transactions on Intelligent Transportation Systems, vol. 10, pp. 1-18, 2021.

[2] M. H. Kashani, M. Madanipour, M. Nikravan, P. Asghari and E. Mahdipour, "A systematic review of IoT in healthcare: Applications, techniques, and trends," Journal of Network and Computer Applications, vol. 192, no. 1, pp. 1-41, 2021.

[3] B. Abidi, A. Jilbab and E. H. Mohamed, "Wireless body area networks: A comprehensive survey," Journal of Medical Engineering Technology, vol. 44, no. 3, pp. 97-107, 2020.

[4] M. S. Hajar, M. O. Al-Kadri and H. K. Kalutarage, "A survey on wireless body area networks: Architecture, security challenges and research opportunities," Computers \& Security, vol. 1, no. 1, pp. 1-27, 2021.

[5] K. Hasan, K. Biswas, K. Ahmed, N. S. Nafi and M. S. Islam, "A comprehensive review of wireless body area network," Journal of Network and Computer Applications, vol. 143, no. 1, pp. 178-198, 2019.

[6] W. Khurshid, M. L. Mat, I. A. Khan, R. Salleh, A. Theodore et al., "Comparative study of congestion notification techniques for hop-by-hop-based flow control in data centre ethernet," IET Networks, vol. 7, no. 4, pp. 248-257, 2018.

[7] W. Khurshid, I. A. Khan, M. L. M. Kiah, O. Khalid and S. A. Madani, "A dynamic threshold calculation for congestion notification in IEEE 802.1qbb," IEEE Communication Letters, vol. 24, no. 4, pp. 744-747, 2020.

[8] Y. Qu, G. Zheng, H. Ma, X. Wang, B. Ji et al., "A survey of routing protocols in WBAN for healthcare applications," Sensors, vol. 19, no. 7, pp. 1-24, 2019.

[9] Q. Nadeem, N. Javaid, S. N. Mohammad, M. Khan, S. Sarfraz et al., "Simple: Stable increased-throughput multi-hop protocol for link efficiency in wireless body area networks," in Eighth Int. Conf. on Broadband and Wireless Computing, Communication, and Applications, Compiegne, France, pp. 221-226, 2013.

[10] N. Javaid, Z. Abbas, M. Fareed, Z. A. Khan and N. Alrajeh, "M-ATTEMPT: A new energy-efficient routing protocol for wireless body area sensor networks," Procedia Computer Science, vol. 19, no. 1, pp. 224-231, 2013.

[11] A. Tauqir, N. Javaid, S. Akram, A. Rao and S. N. Mohammad, "Distance aware relaying energy-efficient: Dare to monitor patients in multi-hop body area sensor networks," in Eighth Int. Conf. on Broadband Wireless Computing, Communication, and Applications, Compiegne, France, pp. 206-213, 2013.

[12] M. R. Jafri, N. Javaid, A. Javaid and Z. A. Khan, "Maximizing the lifetime of multi-chain PEGASIS using sink mobility," World Applied Sciences Journal, vol. 21, no. 9, pp. 1-14, 2013.

[13] N. Javaid, A. Ahmad, Q. Nadeem, M. Imran and N. Haider, "iM-SIMPLE: iMproved stable increasedthroughput multi-hop link efficient routing protocol for wireless body area networks," Computers in Human Behavior, vol. 51, no. 1, pp. 1003-1011, 2015.

[14] F. Jamil, M. A. Iqbal, R. Amin and D. Kim, "Adaptive thermal-aware routing protocol for wireless body area network," Electronics, vol. 8, no. 1, pp. 1-28, 2019.

[15] R. Kaur, B. P. Kaur, R. P. Singla and J. Kaur, "AMERP: Adam moment estimation optimized mobility supported energy efficient routing protocol for wireless body area networks," Sustainable Computing: Informatics and Systems, vol. 31, no. 1, pp. 1-9, 2021.

[16] O. Smail, A. Kerrar, Y. Zetili and B. Cousin, "ESR: Energy aware and stable routing protocol for WBAN networks," in Int. Wireless Communications and Mobile Computing Conference (IWCMC), Paphos, Cyprus, pp. 452-457, 2016.

[17] S. R. Chavva and R. S. Sangam, "An energy-efficient multi-hop routing protocol for health monitoring in wireless body area networks," Network Modeling Analysis in Health Informatics and Bioinformatics, vol. 8, no. 1, pp. 1-10, 2019.

[18] E. H. Mamdani and S. Assilian "Mamdani and sugeno fuzzy inference systems," MathWorks, [Online]. Available: https://www.mathworks.com/help/fuzzy/types-of-fuzzy-inference-systems.html. [Accessed 25 October 2021]. 
[19] F. Aadil, B. Mehmood, N. U. Hasan, S. Lim, S. Ejaz et al., "Remote health monitoring using IoT-based smart wireless body area network," Computers, Materials \& Continua, vol. 68, no. 2, pp. 2499-2513, 2021.

[20] W. R. Heinzelman, A. Chandrakasan and H. Balakrishnan, "Energy-efficient communication protocol for wireless microsensor networks," in Proc. 33rd Annual Hawaii Int. Conf. on System Sciences, Maui, Hawaii, pp. 1-10, 2000.

[21] D. B. Smith, D. Miniutti, T. A. Lamahewa and L. W. Hanlen, "Propagation models for body-area networks: A survey and new outlook," IEEE Antennas and Propagation Magazine, vol. 55, no. 5, pp. 97-117, 2013.

[22] S. Palanisamy, B. Thangaraju, O. I. Khalaf, Y. Alotaibi, S. Alghamdi et al., "A novel approach of design and analysis of a hexagonal fractal antenna array (HFAA) for next-generation wireless communication," Energies, vol. 14, no. 19, pp. 6204-6216, 2021.

[23] M. Geetha and R. Ganesan, "CEPRAN-Cooperative energy efficient and priority based reliable routing protocol with network coding for WBAN," Wireless Personal Communications, vol. 117, no. 4, pp. 31533171, 2021.

[24] T. S. Rappaport, "Mobile Radio Propagation: Large-Scale Path Loss," in Wireless Communications: Principles and Practice, $2^{\text {nd }}$ ed., New York: Pearson, 2002.

[25] R. A. Khan, Q. Xin and N. Roshan, "RK-Energy efficient routing protocol for wireless body area sensor networks," Wireless Personal Communications, vol. 116, no. 1, pp. 709-721, 2021.

[26] S. Bharany, S. Sharma, S. Badotra, O. I. Khalaf, Y. Alotaibi et al., "Energy-efficient clustering scheme for flying Ad-hoc networks using an optimized LEACH protocol," Energies, vol. 14, no. 19, pp. 6016-6026, 2021.

[27] A. F. Subahi, Y. Alotaibi, O. I. Khalaf and F. Ajesh, "Packet drop battling mechanism for energy aware detection in wireless networks," Computers, Materials and Continua, vol. 66, no. 2, pp. 2077-2086, 2020.

[28] K. Takabayashi, H. Tanaka, C. Sugimoto, K. Sakakibara and R. Kohno, "Performance evaluation of a quality of service control scheme in multi-hop WBAN based on IEEE 802.15.6," Sensors, vol. 18, no. 11, pp. 1-26, 2018.

[29] G. Abbas, Z. H. Abbas, S. Haider, T. Baker, S. Boudjit et al., "PDMAC: A priority-based enhanced TDMA protocol for warning message dissemination in VANETs," Multidisciplinary Digital Publishing Institute, vol. 20, no. 1, pp. 1-20, 2020.

[30] X. Wang, G. Zheng, H. Ma, W. Bai, H. Wu et al., "Fuzzy control-based energy-aware routing protocol for wireless body area networks," Journal of Sensors, vol. 2021, pp. 1-13, 2021, https://doi. org/10.1155/2021/8830153.

[31] N. Veeraiah, O. I. Khalaf, C. V. P. R. Prasad, Y. Alotaibi, A. Alsufyani et al., "Trust aware secure energy efficient hybrid protocol for manet," IEEE Access, vol. 9, pp. 120996-121005, 2021.

[32] I. Demirkol, F. Alagoz, H. Deli and C. Ersoy, "Wireless sensor networks for intrusion detection: Packet traffic modeling," IEEE Communications Letters, vol. 10, no. 1, pp. 22-24, 2006. 